\title{
Flexible endoscopic treatment for Zenker's diverticulum: from the lumen to the third space
}

\author{
Roberta Masellia*, Marco Spadaccinia,b*, Annalisa Cappellob, Edoardo Vespa ${ }^{a, b}$, Milena Di Leo ${ }^{a}$, \\ Alessandro Fugazza ${ }^{a}$, Gaia Pellegatta a , Piera Alessia Galtieria, Elisa Chiara Ferrara ${ }^{a}$, Andrea Anderlonia, \\ Silvia Carraraa , Viveksandeep Thoguluva Chandrasekarc, Paul J. Belletruttid ${ }^{\mathrm{d}}$, Alessandro Repicia, ${ }^{\mathrm{a} b}$ \\ Humanitas Clinical and Research Center -IRCCS- Rozzano, Italy; Humanitas University, Rozzano, Italy; Mayo Clinic, \\ Phonix, Arizona, USA; University of Calgary, Canada
}

\begin{abstract}
Zenker's diverticulum (ZD) is a rare outpouching of the esophageal mucosa herniating posteriorly through Killian's triangle. Treatments of ZD aim to dissect the cricopharyngeal muscle to remove the underlying dysfunctional condition. In the last decade, a septotomy performed utilizing a flexible endoscope has been reported as a safe and effective alternative to both open surgery and rigid endoscopic diverticulotomy. More recently, Li et al described a novel endoscopic technique to treat $\mathrm{ZD}$, named "submucosal tunneling endoscopic septum division", inspired by the peroral endoscopic myotomy (POEM) procedure developed for achalasia. Subsequently, the term Z-POEM was introduced and has become the most frequently used acronym to define the tunneling technique for $\mathrm{ZD}$. This article describes the flexible therapeutic endoscopic strategies for treating ZD, including the novel third space approach, which seems to show promising potential in terms of clinical efficacy and safety.
\end{abstract}

Keywords Endoscopy, esophagus, Zenker's diverticulum, third space, peroral endoscopic myotomy

Ann Gastroenterol 2021; 35 (2): 1-6

\section{Introduction}

Zenker's diverticulum (ZD) is a pulsion diverticulum deriving from the herniation of both the mucosa and the submucosa of the posterior pharyngeal wall through Killian's triangle, a locus minoris resistentiae situated between the oblique

aEndoscopy Unit, Humanitas Clinical and Research Center -IRCCS-, Rozzano, Italy (Roberta Maselli, Marco Spadaccini, Edoardo Vespa, Milena Di Leo, Alessandro Fugazza, Gaia Pellegatta, Piera Alessia Galtieri, Elisa Chiara Ferrara, Andrea Anderloni, Silvia Carrara,

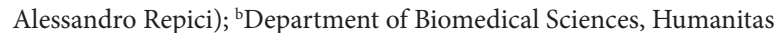
University, Rozzano, Italy (Marco Spadaccini, Annalisa Cappello,

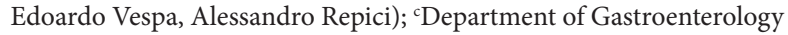
and Hepatology, Mayo Clinic, Phoenix, Arizona, USA (Viveksandeep Thoguluva Chandrasekar); ${ }^{\mathrm{d} D i v i s i o n}$ of Gastroenterology and Hepatology, University of Calgary, Calgary, Canada (Paul J. Belletrutti)

${ }^{*}$ Contributed equally to this work

Conflict of Interest: None

Correspondence to: Marco Spadaccini, MD, Digestive Endoscopy Unit, Via Manzoni 56, 20089 Rozzano, Milan, Italy,

e-mail: marcospadaccini9@gmail.com

Received 21 April 2020; accepted 15 October 2020; published online 16 January 2021

DOI: https://doi.org/10.20524/aog.2021.0575 muscle fibers of the lower pharyngeal constrictor muscle (thyropharyngeus) and its transverse fibers, the so-called cricopharyngeal muscle (CM) [1]. The CM itself, together with the intrinsic musculature of the upper esophagus, forms the upper esophageal sphincter (UES). The pathogenesis of ZD is not completely understood; however, it appears to be related to impaired UES compliance [2]: the altered relaxation of the $\mathrm{CM}$ during swallowing causes an inadequate clearance of the bolus from the hypopharynx, leading to increased intraluminal pressure and the development of a ZD over time [3].

$\mathrm{ZD}$ is the most common hypopharyngeal diverticulum, with an estimated prevalence of $0.01-0.11 \%$. Since the number of asymptomatic patients is unknown [4], the true prevalence is likely to be significantly higher. It is more frequently diagnosed in the "western world" (Northern Europe, the United States, Canada and Australia), with a prevalence in males in the 7th and 8th decades of life, while it is a very rarely reported finding in Eastern countries [4].

The major symptom is dysphagia, which occurs in 80$90 \%$ of patients. Other reported symptoms are regurgitation, foreign body sensation, halitosis, chronic cough and systemic complications such as weight loss, and aspiration pneumonia.

Treatment is indicated for symptomatic ZD [5]. The treatment of $\mathrm{ZD}$ aims to transect the $\mathrm{CM}$ to eliminate the septum between the diverticulum and the upper esophagus, thus relieving the dysfunctional condition. Currently there are 3 
main therapeutic approaches for dealing with symptomatic ZD: open trans-cervical diverticulectomy [6], and cricopharyngeal septotomy using either a rigid [7] or a flexible endoscope. The decision as to whether to use an open or a transoral approach is based on weak recommendations and low-quality evidence, given the lack of high-quality comparative studies [5]. In daily practice, it depends on several factors, including the anatomy and size of the diverticulum, the patient's current health status, comorbidities, patient preference, and local expertise.

Transcervical diverticulectomy is an effective option, with both a low risk of technical failure and a low recurrence rate [6]; however, septotomy performed through a rigid endoscope has been shown to achieve comparable efficacy outcomes [8], resulting in quicker diet resumption, lower adverse event rates and a shorter inpatient stay [8-11].

Nevertheless, approaches using rigid endoscopy have several limitations, such as the need for general anesthesia and significant rates of intraoperative failure (5-10\%). Small diverticular size $(<3 \mathrm{~cm})$, inadequate jaw opening and restricted neck mobility are the main causes of technical failure $[10,11]$. During the last few decades, in an effort to overcome such limitations, flexible endoscopic approaches have been established as safe and effective alternatives to both open surgery and the rigid endoscopic treatments [12]. This paper aims to provide a comprehensive point of view on flexible endoscopic therapies for ZD, from the "classic" flexible endoscopic septum division (FESD), to the most cutting-edge third space approaches.

\section{Luminal flexible endoscopic approaches}

FESD (Fig. 1A) was first described by Mulder and Ishioka $[13,14]$ more than 20 years ago. It shares the same principles and rationale as rigid endoscopy: it involves a full thickness incision of the mucosa, submucosa and the muscular fibers that form the diverticular septum. By cutting the entire septum and creating a common cavity between the esophagus and diverticulum, a myotomy is automatically performed (Fig. 2).

\section{Procedure}

Differences in the sedation approach to these patients have been recorded in the published papers. Several authors still prefer to perform FESD under general anesthesia [15], with many others preferring propofol-based deep sedation in the majority of patients [16].

The procedure is performed with the patient in the left lateral decubitus position. No prophylactic antibiotic therapy is required. It begins with initial endoscopic examination of the pouch and suction of any retained material from the diverticulum. Prior to performing the procedure, it is common practice to introduce a nasogastric/orogastric tube via a guidewire that has been advanced under endoscopic guidance into the gastric cavity. It is useful to constantly recognize the esophageal lumen by visualizing this tube during myotomy [17]. Less commonly, instead of the orogastric tube, some endoscopists use a soft diverticuloscope (ZD overtube, ZDO-22-30; Cook Medical, Winston-Salem, North Carolina), placed as an overtube in the hypopharynx, to stabilize and visualize the septum. Even though the diverticuloscope might potentially improve safety and maneuverability, there are no significant differences in clinical outcomes between the different approaches [18-21].

A distal attachment, such as a standard short transparent cap, is also frequently used to enhance visualization, improve endoscope stability, and gently stretch the septum at the time of its incision. In addition to the different accessories that can be selected to improve septum exposure, varying cutting techniques and devices can be used (needle-knife, hook-knife, monopolar forceps, argon plasma coagulation) depending on the endoscopist's training and personal experience, with the most commonly used devices being the hook knife and the needle knife (Olympus medical, Tokyo, Japan) (Electrocautery settings: Endocut 1.0, Forced coag 4.0) [19,21-25]. Submucosal injection is not required before septotomy. After septotomy, one or more endoclips (usually up to 3 clips) are routinely placed to close the incision to reduce the likelihood of delayed perforation or bleeding.

Additional endoscopic approaches have been described, involving harmonic scalpels, stapling devices, the stag beetle knife (SB Knife, Sumitomo Bakelite, Tokyo, Japan), and polypectomy snares [26-28]. However, they are still anecdotal.

Clinical follow up is indicated and postprocedural radiography via barium esophagography or computed tomography scan is not advised, unless there is persistence or recurrence of symptoms.

\section{Outcomes}

Available data from published series have reported safety and efficacy using various techniques and devices, with a high rate of durable symptom relief ( $>90 \%$ ) and a low rate of diverticular recurrence. In 2016, Ishaq et al [29] investigated outcomes of FESD by pooling data from the available series, reporting a success rate of $91 \%$ with a recurrence rate of $11.6 \%$. However, most of these studies were retrospective, and comparison among different studies is biased because there is no consensus as to the definition of post-procedural clinical success.

In terms of safety, a pooled adverse event rate of $13 \%$, mainly due to perforation (7\%) and bleeding (5\%), was reported by a more recent meta-analysis [30], in line with the previous findings by Ishaq et al [29]. Rarer adverse events, including pneumonia, fever, subcutaneous emphysema and neck abscess formation, were also reported. The risk of complications does not appear to be related to either clinical (ZD size or previous treatments) or technical aspects, such as the type of sedation or the cutting device used [29,31].

There are no studies comparing the open (surgical) approach and flexible endoscopic treatment. However, FESD efficacy and 

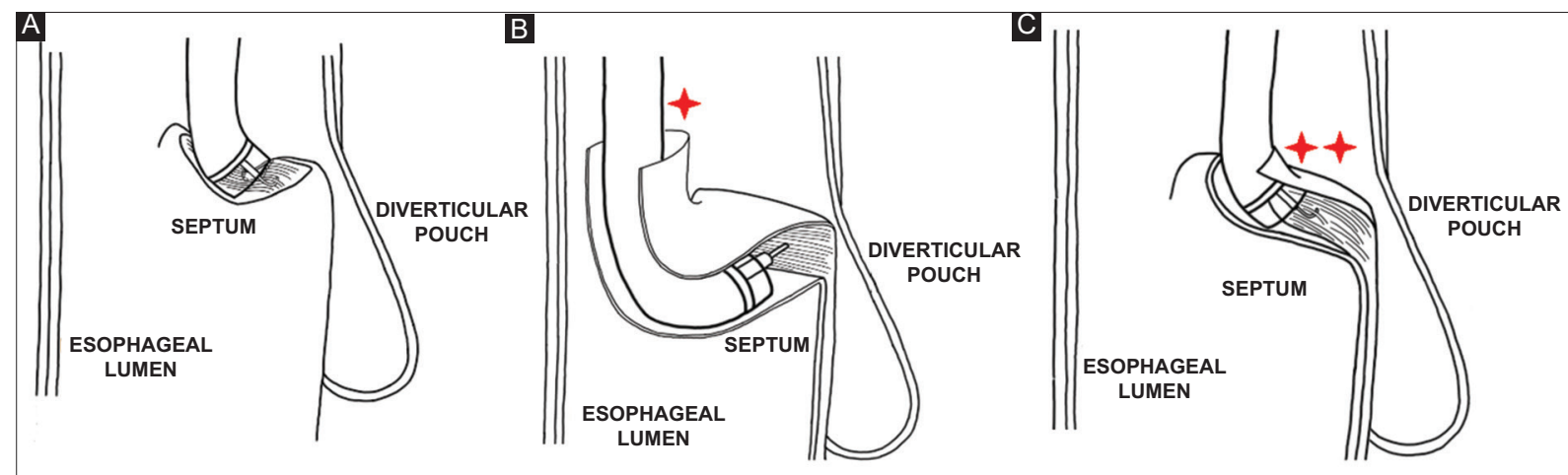

Figure 1 Flexible endoscopy options: (A) Flexible endoscopic septum division, (B) Zenker peroral endoscopic myotomy (Z-POEM), (C) Peroral endoscopic septotomy (POES)

${ }^{*}$ Submucosal access in Z-POEM is located proximally to the septum, in the hypopharynx. ${ }^{* *}$ Submucosal access in POES is located at the top of the septum

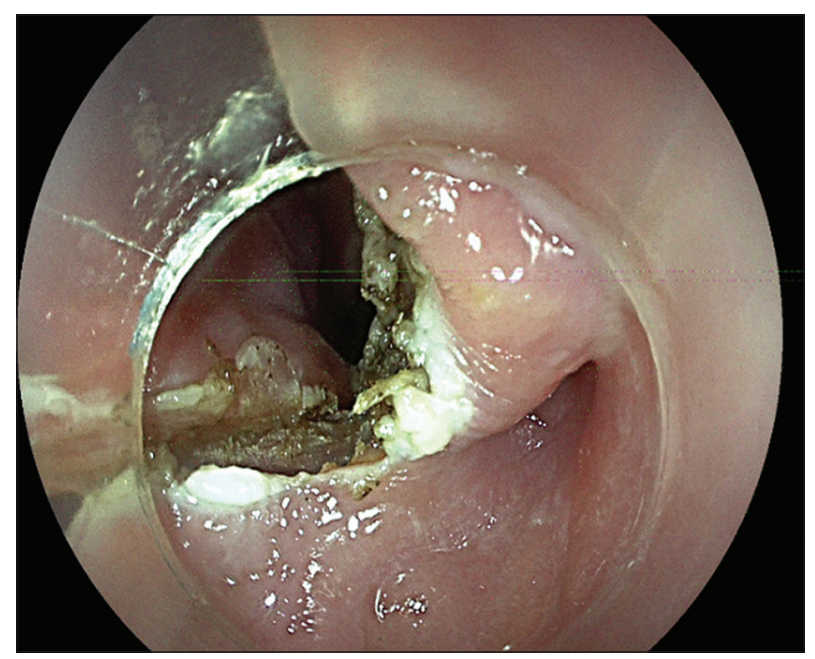

Figure 2 Endoscopic view after complete septotomy by flexible endoscopic septum division approach

safety outcomes were shown to be comparable to those achieved by transoral septotomy with a rigid endoscope [16,32], yet with a less invasive approach. As a matter of fact, it can be performed without general anesthesia and does not require neck hyperextension (as is necessary for the rigid endoscopic procedure) $[13,24]$. Consequently, the procedure can be safely performed in the gastrointestinal endoscopy suite, even in an outpatient setting [16].

The need for general anesthesia and the high rate of intraoperative abandonment owing to restricted neck mobility, combined with comparable success and adverse events rates have led the European Society of Gastrointestinal Endoscopy (ESGE) to recommend the use of FESD as the first-line therapy for ZD [5].

\section{Third-space flexible endoscopic approaches}

Recent advances in natural orifice transluminal endoscopic surgery (NOTES) have given rise to novel myotomy techniques [33]. Recently, Li et al described a novel endoscopic technique to treat $\mathrm{ZD}$, named "submucosal tunneling endoscopic septum division" [34], inspired by the peroral endoscopic myotomy (POEM) procedure developed for achalasia [35]. Subsequently, the term Z-POEM was introduced by Hernández Mondragón et al [36] and was adopted in further studies as the preferred acronym to define the tunneling technique for ZD (Fig. 1B).

The theoretical advantage of this approach is that it can more readily perform a complete transection of the entire muscular septum by operating within a submucosal tunnel, thus maintaining the mucosal integrity (Fig. 3). This procedure has the potential to reduce the risk of perforation, mediastinitis and recurrence [34].

As with the FESD technique, the procedure is performed with the patient in the left lateral decubitus position. No prophylactic antibiotic therapy is required. Submucosal injection and subsequent longitudinal mucosotomy are performed $3 \mathrm{~cm}$ proximal to the septum as the tunnel entry. A submucosal tunnel is then created along both sides of the septum, ending 1-2 cm distal to the bottom of the diverticulum, in order to have an adequate endoscopic view of the entire muscular septum. The CM fibers of the septum are then transected down to the bottom of the diverticulum and further into the normal esophageal muscle. The mucosal incision site is finally sealed with up to 3 clips.

After preliminary reports [34-37], Yang et al [38] collected data from an international multicenter cohort showing a promising clinical success rate of $92 \%$ with a perforation rate of $5.5 \%$. Even if a direct comparison with standard FESD is lacking, a mean procedural time of around $50 \mathrm{~min}$ is undoubtedly longer compared to the standard approach. This was perhaps to be expected, considering that all participating centers shared their initial Z-POEM cases at the beginning of their learning curve. However, these data were confirmed by the first comparison study presented at the 2020 Digestive Diseases Week by Aslan et al [39]. Still, retrospectively matching 20 FESDs and 9 Z-POEMs might not be considered a reliable way to corroborate the strength of a third space approach; thus, future studies are needed. 


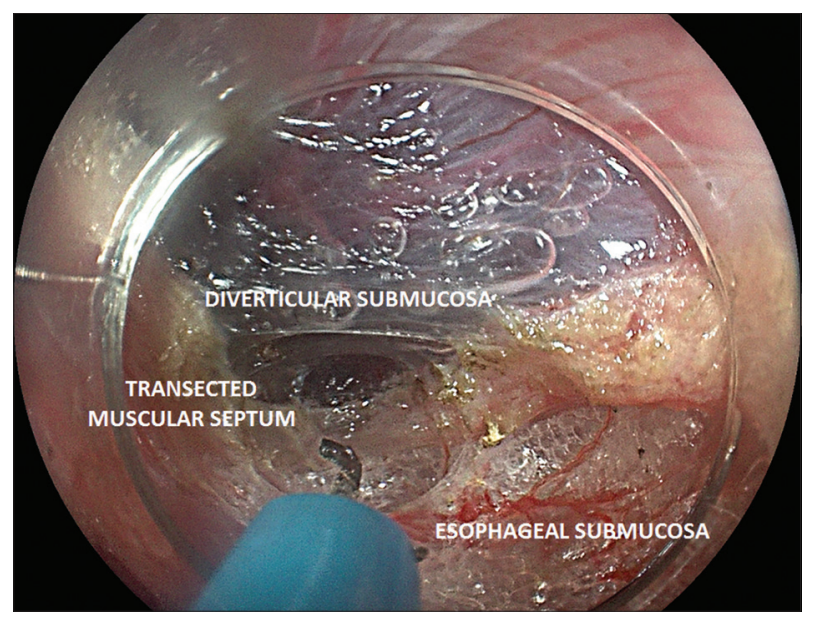

Figure 3 Endoscopic view after complete myotomy by Zenker peroral endoscopic myotomy (Z-POEM) approach (intra-tunnel view)

More recently, Repici et al [40] conceived of an alternative third space approach, called peroral endoscopic septotomy (POES), to overcome the technical challenges of the Z-POEM technique. In the "standard" Z-POEM approach, the initial mucosotomy has to be created approximately $1-3 \mathrm{~cm}$ proximal to the septum, at the boundaries between the pharynx and the UES. In this area, muscular spasm as well as anatomical limitations may reduce the ability to properly open and close the mucosal incision. Hence, in order to gain direct access to the ZD muscular septum without the need of long tunneling starting at pharyngeal level, they proposed to perform the mucosal cut alongside the long axis of the septum, and directly on top of it [40,41] (Fig. 4).

Unlike the "classic" Z-POEM, a procedural time of 13.8 \pm 5.1 min seems to be comparable with standard FESD. Further, a clinical success rate of $95 \%$ with no intra- or postprocedural adverse events and only 4 instances of mild asymptomatic subcutaneous emphysema were reported. The procedure was performed on 16 of 20 patients in an outpatient setting with same-day discharge. Overall, the mean hospital stay was $1.2 \pm 0.4$ days.

\section{Discussion}

The flexible endoscopic approach is recommended over surgery (transcervical diverticulectomy and rigid endoscopic septotomy) by current guidelines [5]. The next step will be the understanding of the clinical indication for each technique. Currently, there are no randomized trials or long-term studies to determine which is the most effective procedure and which one is durable. This is directly reflected in the lack of highquality, strong recommendations for the management of $\mathrm{ZD}$. For instance, in the choice of best therapeutic approach the most important factor is the experience of the operator (both surgeon and endoscopist). Unfortunately, the low incidence of the condition makes it more difficult to conduct a large-scale study and therefore to personalize the therapeutic approach in ZD.

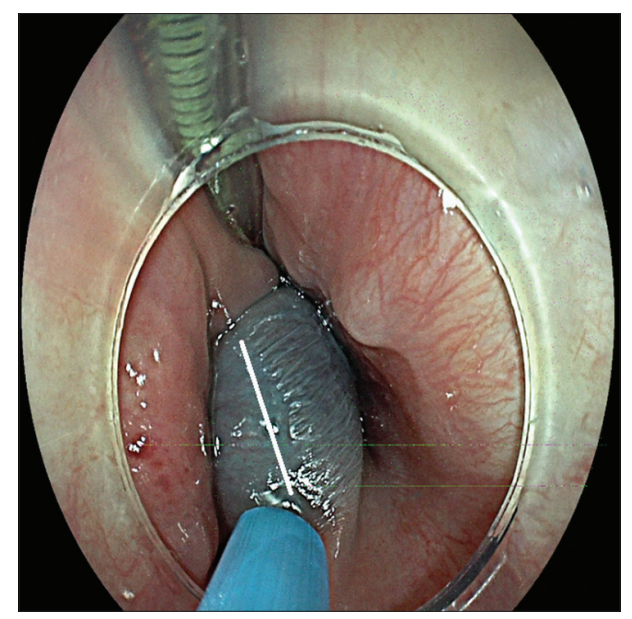

Figure 4 Mucosal incision at the top of the septum for third-space access during peroral endoscopic septotomy (POES) approach

Concerning the FESD technique, both the efficacy and the safety seems to be related to a simple question: how much to cut? Unfortunately, this topic is still difficult to standardize, mainly because of the lack of unequivocal anatomic landmarks. The only advice to be given is to reach the end of the pouch in order to have a unique cavity along with the esophageal lumen. Of course, third space approaches bypassed this problem by taking advantage of a tunneling technique. This permits the performance of longer myotomies without the fear of serious complications related to myotomy extension.

On the other hand, several issues are still open regarding tunneling techniques. First of all, the anatomical site is quite different from the distal esophagus where POEM was developed. The narrower location and the lack of an external muscular layer at the hypopharyngeal site may lead to a more challenging procedure with the risk of serious adverse events and a longer learning curve. However, in the first reports no cases of mediastinitis were reported. Secondly, third space approaches involve dissection of the CM, leaving the pouch sac unchanged. In large diverticula, the remnant mucosal excess might potentially result in a pseudo-diverticulum that could theoretically lead to persistence of symptoms. Of course, in the initial experience by Yang et al [38] no sizelimits were applied, yet the treatment still showed promising efficacy. Thus, hopefully future evidence will rule out this potential drawback, eliminating the clinical relevance of the mucosal excess in larger diverticula. With this in mind, in Fig. 5 we have summarized the difference instances in an attempt to outline a hypothetical flowchart, as evidence-based as possible, to help the reader's orientation in this evolving scenario.

In conclusion, flexible endoscopic approaches are effective and safe options for managing ZD. Data on third space techniques are promising as regards the further improvement in patient outcomes; however, further studies are required to define which patients could benefit the most from such an approach. 


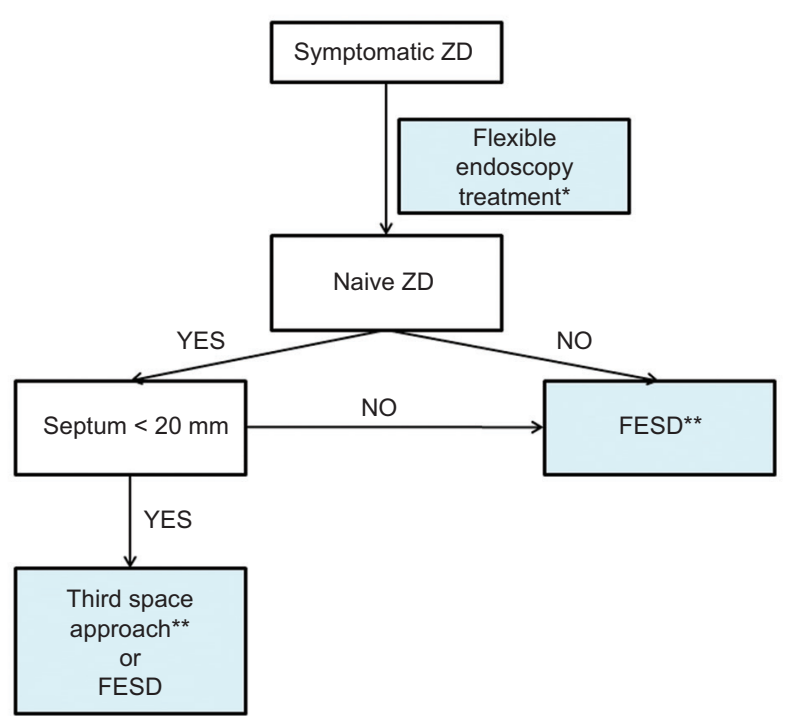

Figure 5 How to deal with different flexible endoscopy options ${ }^{*}$ According to ESGE guidelines, flexible endoscopy should be considered as the first option for symptomatic patients with Zenker's diverticulum (ZD). Surgical and rigid endoscopy treatment may be viable alternatives. ${ }^{* *}$ Data on recurrent $\mathrm{ZD}$ are still scanty for thirdspace approaches. In the future this could change. ${ }^{\star *}$ For large ZD a third space approach might leave a significant mucosal flap. We still do not know the clinical consequence of such mucosal excess, however a reliable and well-known procedure such as flexible endoscopic septum division (FESD) may be preferre

\section{References}

1. Zenker FA, von Ziemssen H. Krankheiten des oesophagus. In: von Ziemssen H, ed. Handbuch der Speciellen. Pathologie and Therapie 1877;7(Suppl) Leipzig: FCW Vogel.

2. Shaw DW, Cook IJ, Jamieson GG, Gabb M, Simula ME, Dent J. Influence of surgery on deglutitive upper oesophageal sphincter mechanics in Zenker's diverticulum. Gut 1996;38:806-811.

3. Cook IJ, Gabb M, Panagopoulos V, et al. Pharyngeal (Zenker's) diverticulum is a disorder of upper esophageal sphincter opening. Gastroenterology 1992;103:1229-1235.

4. Watemberg S, Landau O, Avrahami R. Zenker's diverticulum: reappraisal. Am J Gastroenterol 1996;91:1494-1498.

5. Weusten BLAM, Barret M, Bredenoord AJ, et al. Endoscopic management of gastrointestinal motility disorders - part 2: European Society of Gastrointestinal Endoscopy (ESGE) Guideline. Endoscopy 2020;52:600-614.

6. Yuan Y, Zhao YF, Hu Y, Chen LQ. Surgical treatment of Zenker's diverticulum. Dig Surg 2013;30:207-218.

7. Lang RA, Spelsberg FW, Winter H, Jauch KW, Hüttl TP. Transoral diverticulostomy with a modified Endo-Gia stapler: results after 4 years of experience. Surg Endosc 2007;21:532-536.

8. Howell RJ, Giliberto JP, Harmon J, et al. Open versus endoscopic surgery of zenker's diverticula: a systematic review and metaanalysis. Dysphagia 2019;34:930-938.

9. Bonavina L, Bona D, Abraham M, Saino G, Abate E. Long-term results of endosurgical and open surgical approach for Zenker diverticulum. World J Gastroenterol 2007;13:2586-2589.

10. Keck T, Rozsasi A, Grün PM. Surgical treatment of hypopharyngeal diverticulum (Zenker's diverticulum). Eur Arch Otorhinolaryngol 2010;267:587-592.

11. Chang CY, Payyapilli RJ, Scher RL. Endoscopic staple diverticulostomy for Zenker's diverticulum: review of literature and experience in 159 consecutive cases. Laryngoscope 2003;113:957-965.

12. Repici A, Pagano N, Fumagalli U, et al. Transoral treatment of Zenker diverticulum: flexible endoscopy versus endoscopic stapling. A retrospective comparison of outcomes. Dis Esophagus 2011;24:235-239.

13. Mulder CJ, den Hartog G, Robijn RJ, Thies JE. Flexible endoscopic treatment of Zenker's diverticulum: a new approach. Endoscopy 1995;27:438-442.

14. Ishioka S, Sakai P, Maluf Filho F, Melo JM. Endoscopic incision of Zenker's diverticula. Endoscopy 1995;27:433-437.

15. Aiolfi A, Scolari F, Saino G, Bonavina L. Current status of minimally invasive endoscopic management for Zenker diverticulum. World J Gastrointest Endosc 2015;7:87-93.

16. Repici A, Pagano N, Fumagalli U, et al. Transoral treatment of Zenker diverticulum: flexible endoscopy versus endoscopic stapling. A retrospective comparison of outcomes. Dis Esophagus 2011;24:235-239.

17. Perbtani Y, Suarez A, Wagh MS. Techniques and efficacy of flexible endoscopic therapy of Zenker's diverticulum. World J Gastrointest Endosc 2015;7:206-212.

18. Rabenstein T, May A, Michel J, et al. Argon plasma coagulation for flexible endoscopic Zenker's diverticulotomy. Endoscopy 2007;39:141-145.

19. Costamagna G, Iacopini F, Tringali A, et al. Flexible endoscopic Zenker's diverticulotomy: cap-assisted technique vs. diverticuloscope-assisted technique. Endoscopy 2007;39:146-152.

20. Mulder CJ. Zapping Zenker's diverticulum: gastroscopic treatment. Can J Gastroenterol 1999;13:405-407.

21. Sakai P, Ishioka S, Maluf-Filho F, Chaves D, Moura EG. Endoscopic treatment of Zenker's diverticulum with an oblique-end hood attached to the endoscope. Gastrointest Endosc 2001;54:760-763.

22. Repici A, Pagano N, Romeo F, et al. Endoscopic flexible treatment of Zenker's diverticulum: a modification of the needle-knife technique. Endoscopy 2010;42:532-535.

23. Ishioka S, Sakai P, Maluf Filho F, Melo JM. Endoscopic incision of Zenker's diverticula. Endoscopy 1995;27:433-437.

24. Case DJ, Baron TH. Flexible endoscopic management of Zenker diverticulum: the Mayo Clinic experience. Mayo Clin Proc 2010;85:719-722.

25. Vogelsang A, Preiss C, Neuhaus H, Schumacher B. Endotherapy of Zenker's diverticulum using the needle-knife technique: long-term follow-up. Endoscopy 2007;39:131-136.

26. Ramchandani M, Nageshwar Reddy D. New endoscopic "scissors" to treat Zenker's diverticulum (with video). Gastrointest Endosc 2013;78:645-648.

27. Battaglia G, Antonello A, Realdon S, Cesarotto M, Zanatta L, Ishaq S. Flexible endoscopic treatment for Zenker's diverticulum with the SB Knife. Preliminary results from a single-center experience. Dig Endosc 2015;27:728-733.

28. Goelder SK, Brueckner J, Messmann H. Endoscopic treatment of Zenker's diverticulum with the stag beetle knife (sb knife) feasibility and follow-up. Scand J Gastroenterol 2016;51:1155-1158.

29. Ishaq S, Hassan C, Antonello A, et al. Flexible endoscopic treatment for Zenker's diverticulum: a systematic review and meta-analysis. Gastrointest Endosc 2016;83:1076-1089.

30. Li LY, Yang YT, Qu CM, et al. Endoscopic needle-knife treatment for symptomatic esophageal Zenker's diverticulum: A metaanalysis and systematic review. J Dig Dis 2018;19:204-214.

31. Sakai P. Evolving flexible endoscopic treatment of Zenker's diverticulum. Gastrointest Endosc 2019;89:887-888.

32. Crawley B, Dehom S, Tamares S, et al. Adverse events after rigid and flexible endoscopic repair of Zenker's diverticula: a systematic review and meta-analysis. Otolaryngol Head Neck Surg 2019;161:388-400. 
33. Brewer Gutierrez OI, Ichkhanian Y, Spadaccini M, Vosoughi K, Repici A, Khashab MA. Zenker's diverticulum per-oral endoscopic myotomy techniques: changing paradigms. Gastroenterology 2019;156:2134-2135.

34. Li QL, Chen WF, Zhang XC, et al. Submucosal tunneling endoscopic septum division: a novel technique for treating Zenker's diverticulum. Gastroenterology 2016;151:1071-1074.

35. Inoue H, Minami H, Kobayashi Y, et al. Peroral endoscopic myotomy (POEM) for esophageal achalasia. Endoscopy 2010;42:265-271.

36. Hernández Mondragón OV, Solórzano Pineda MO, Blancas Valencia JM. Zenker's diverticulum: Submucosal tunneling endoscopic septum division (Z-POEM). Dig Endosc 2018;30:124.

37. Delis K, Robotis J, Sachitzi E, Mastronikola E. Submucosal tunneling endoscopic septum division of a Zenker's diverticulum. Ann Gastroenterol 2018;31:634.
38. Yang J, Novak S, Ujiki M, et al. An international study on the use of peroral endoscopic myotomy in the management of Zenker's diverticulum. Gastrointest Endosc 2020;91:163-168.

39. Aslan F, Yilmaz O, Sengun B, et al. Tu1951 a new technique in treatment of zenker diverticulum: submucosal tunneling endoscopic septum division (z-poem) versus classic endoscopic septomyotomy techniques. Gastrointest Endosc 2019;89(6 Suppl):AB629. doi:10.1016/j.gie.2019.03.1095

40. Repici A, Spadaccini M, Belletrutti PJ, et al. Peroral endoscopic septotomy for short-septum Zenker's diverticulum. Endoscopy 2020;52:563-568.

41. Mavrogenis G, Tsevgas I, Zachariadis D, Bazerbachi. Mucosotomy at the top of the septum facilitates tunneling and clipping during peroral endoscopic myotomy for Zenker's diverticulum (Z-POEM). Ann Gastroenterol 2020;33:101. 